Correction

\title{
Minardi, E. Thermodynamics of High Temperature Plasmas. Entropy, 2009, 11, 124-221
}

\section{Ettore Minardi}

Istituto di Fisica del Plasma, Via R. Cozzi 53 I-20125 Milano Italy; E-Mail: minardi@ifp.cnr.it

Received: 4 September 2009 / Accepted: 14 September 2009 / Published: 14 September 2009

I discovered some typographical errors in the paper "Thermodynamics of High Temperature Plasmas" [1] and some points that need clarification. These defects are remedied in this correction.

Page 127, $13^{\text {th }}$ line from below:

..., which selects the ...

Page 127, $12^{\text {th }}$ line from below:

...which plays...

Page 139, line after Equation (6.7);

Where one equality is a consequence of the other when the plasma is stationary $\left(\vec{\partial}_{p} / \partial=0\right)$.

Page 139, Equation (6.8) should be corrected as follows:

$$
\frac{E_{e x t}}{\mu^{2}}\left(\nabla^{2} j_{p}+\mu^{2} j_{p}\right)=-F
$$

Page 142: the paragraph between Equations (8.4) and (8.5) should be replaced as follows:

In accordance with (8.4), the term $\varphi$ is generated by the external heating described by $p_{E}$. In addition $\varphi$ includes the harmonic function $C \ln (r / a),(C=c o n s t)$, which arises from the presence of the sawteeth zone and its boundary conditions. Indeed, it will be shown in Section 20, Equation (20.7), that this function is related to the heat balance between the sawteeth zone and the relaxed zone where the SME equation holds. Thus, applying (8.4), the last term of (8.3) can be written as $\int_{\Omega} \varphi E_{\text {ext }} d \Omega$, which describes the work of the electric field on that part of the current density, which is related to the auxiliary electron heating of the relaxed zone through the SME equation. It will be shown in 
Section 17 that the effect of the auxiliary heating becomes negligible under the same conditions that insure the profile consistency in the tokamak, namely in the limit $\mu a \rightarrow 0$. Thus, under these conditions (to be presented in chapter 4), and in the absence of sawteeth $(C=0)$, only the electromagnetic effects described by the Poynting term are present in the expression of $d \Phi_{\text {int }} / d t$ and $d \Phi_{\text {int }} / d t=0$ when the plasma is isolated. For the expression of $d \Phi_{\text {int }} / d t$ in the presence of sawteeth $(C \neq 0)$ see section 18 .

In the case of a force free equilibrium, which satisfies the equations

$$
\vec{j}=\frac{\mu c}{4 \pi} \vec{B}=\frac{\mu c}{4 \pi} \nabla \times \vec{A}_{p}=\frac{c}{4 \pi} \nabla \times \vec{B}
$$

the integrand...

Page 144: $5^{\text {th }}$ line from above:

...will be justified in section 17 . In this case, assuming for simplicity the absence of the sawteeth zone ( $C=0$ ), Equation (8.4) becomes...

Page 145, $5^{\text {th }}$ line from above:

...modeled by taking for the safety factor the expression

$$
q(x)=q_{0}+\left(1-q_{0}\right)(x / \lambda)^{2}
$$

A JET-like...

Page 145, $9^{\text {th }}$ line from above up to Table I included:

We take 1.5MA for the total current and 3.3T for the toroidal field. The results are independent of the parameter $\mu a$ as far as $\mu a \rightarrow 0$ (see section 17 for the physical meaning of this limit). The location of the $q(\lambda)=1$ surface is taken at $\lambda=0.16$ and the total current is kept fixed. The current density and the poloidal field across the $q(\lambda)=1$ surface are continuous. The quantity $\Delta \psi$ denotes the variation of the poloidal flux with respect to an initial state with $\Delta \psi=0$. In Table I, $\Delta \psi$ is the loss of poloidal flux (divided by $2 \pi$ ), $q_{0}$ is the value of the safety factor on the minor axis, $j(\lambda), j(1)$ are the values of the current density at the inner and outer border respectively, and $|\tau| \Delta S_{p}$ given by (9.7) is the electromagnetic energy released by the collective system when the electromagnetic entropy increases.

Figure 1 shows the evolution of the current density profiles when the parameters of Table I are varying. One observes that the increase of entropy and the concomitant release of electromagnetic energy are accompanied by loss of poloidal flux, by the increase of the current density on axis and by a pronounced increase of the current density at the border together with a decrease near the $q=1$ surface. Thus, the states with higher entropy are those with lower values of $q_{0}$, which fact explains why a sawteeth zone with $q_{0}<1$ is always formed in tokamaks (unless the sawteeth are contrasted by some external action). 
Table I. Meaning of symbols: $\Delta \psi$, variation of the poloidal flux (divided by $2 \pi$ ); $q_{0}$, safety factor on magnetic axis; $j(\lambda)$, current density on the $q=1$ surface; $j(1)$, current density at the outer border of the relaxed region; $\Delta \Phi_{\text {int }}$, electromagnetic energy released associated with the magnetic entropy increase $\Delta S_{p}$.

\begin{tabular}{lllll}
\hline$\Delta \psi$ & $q_{0}$ & $j(\lambda)$ & $j(1)$ & $|\tau| \Delta S_{p}$ \\
$T-m^{2}$ & & $M A / m^{2}$ & $M A / m^{2}$ & $M J$ \\
\hline 0.00 & 0.98 & 1.72 & 0.022 & 0.00 \\
-0.017 & 0.92 & 1.62 & 0.057 & 0.030 \\
-0.038 & 0.87 & 1.52 & 0.088 & 0.059 \\
-0.051 & 0.81 & 1.41 & 0.12 & 0.090 \\
-0.063 & 0.77 & 1.34 & 0.15 & 0.11 \\
\hline
\end{tabular}

When the system...

Page 146, in the caption of fig.1 replace "outgoing poloidal flux" by

"loss of the poloidal flux"

Page 146, the line after Equation (10.1):

$\ldots$ one has for $\delta S_{p}$ (where $S_{p}$ is given by (6.3)):

Page 147, the line after Equation (10.5):

$\ldots$ is also a solution of (10.5) and...

Page 148, $18^{\text {th }}$ line from above:

Replace "paragraphs” by “sections”.

Page 149, $14^{\text {th }}$ line from above:

Replace “\$1” by “Section 1”.

Page 150, the following equality should be added to the equalities (11.5):

$d \alpha^{i}=\vec{\xi} \cdot \nabla \alpha^{i}$

Page 157, 3th line from above:

Consequence of the singularity of $\mu_{n}^{2} \propto-\tau_{n}^{-1}$ at $r=s$. For getting...

Page 161: the argument about the minimization of the poloidal magnetic energy based on Equation (17.2) is insufficient. However the minimization still holds, but the paragraphs starting with "For seeing the physical meaning..." up to the first five lines of page 162 must be replaced as follows:

For seeing the physical meaning of this fact let us consider the poloidal magnetic energy in the relaxed region $\lambda s a \leq r \leq s a$ : 


$$
w=\frac{1}{2 c} \int_{\Omega} j_{\phi} A_{\phi} d \Omega=-\frac{1}{2 c R} \int_{\Omega} j_{\phi} \psi d \Omega
$$

For a given set of external parameters (see Table II) $w$ depends on the safety factor on axis $q_{0}$ (the safety factor in the sawteeth region is modeled according to (9.11)), on the position $\lambda$ sa of the $q=1$ surface, on the parameter $\mu a$ and on the value $j(s a)$ of the current density at the outer border of the relaxed region. The average values of $q_{0}$ and of $\lambda s a$ are determined by the dynamics of the sawteeth in the zone $q<1$. Thus we keep these parameters as fixed and calculate $w$ as function of $\mu a$ keeping fixed also the total current, while the current density at the border is varying. One finds that $w$ decreases when $\mu a$ decreases, while the current density at the border increases. Both quantities reach asymptotically a constant value for $\mu a \rightarrow 0$. Therefore the limiting process $\mu a \rightarrow 0$ with constant total current minimizes the poloidal magnetic energy (17.1). This fact is at the root of the profile consistency of the SME states (the term "profile consistency" has been used for the first time by Coppi).

The role played by the parameter....

Page 165, the first two lines of this page should be replaced as follows:

But the minimization principle of the poloidal magnetic energy tells us (Section 17) that $\mu a \rightarrow 0$. In this case, Equation (16.3) gives $\vec{j}_{p}-\left(\mu^{2} c / 4 \pi\right) \vec{A}_{p}=D_{0} \ln x+D_{0} \rightarrow \vec{j}_{p}$ where $\vec{j}_{p}$ is the solution of the SME equation for $\mu a \rightarrow 0$. Then one has from (18.16) that $d \Phi_{\text {int }} / d t \rightarrow \int_{\Omega} j_{p} E_{e x t} d \Omega$ and, contrary to Equation (8.3) the Poynting radiation term is absent in the expression for $d \Phi_{\text {int }} / d t$.

This result...

Page 165, seven lines from above:

describes the Poynting flux, should be replaced by includes the Poynting flux..

Page 165, the equation (19.2) should be corrected as follows:

$$
j_{\phi}=-\frac{c R}{4 \pi}\left(\frac{\left(F^{2}(\psi)\right)^{\prime}}{2 R^{2}}+4 \pi p(\psi)^{\prime}\right)
$$

Page 171: the paragraph comprised between Equation(20.6) and the line after Equation(20.7) must be modified as follows:

$$
q_{h}=-\frac{E_{e x t}}{\mu^{2}}\left(\frac{d j}{d r}-\frac{D_{0}}{r}\right) .
$$

where $D_{0}$ is the same as in (16.3), as required by consistency with the power balance. Thus, recalling (7.2), the heat flux across the surface $S=4 \pi^{2} r R$ becomes (neglecting non inductive currents) 


$$
q_{h} S=-\frac{E_{e x t}}{\mu^{2}}\left(\frac{d j}{d r}-\frac{D_{0}}{r}\right) S=4 \pi^{2} R \int_{0}^{r}\left(E_{e x t} j+p_{E}\right) r d r
$$

The heat flux is continuous across the surface $\hat{q}=1$ (we note that...

Page 171, eight lines from below: the expression for $q(x)$ should be corrected as follows: $q(x)=q_{0}+\left(1-q_{0}\right)(x / \lambda)^{2}$ for the safety factor..

Page 171, the equality in the line after Equation (20.8) should be corrected as follows: Here $\hat{v}^{2}=-(4 \pi R / c)(d j / d r)_{\lambda s a}(d r / d \psi)_{\lambda s a}$.

Page 172, the expression for $A\left(Z_{\text {eff }}\right)$ should be corrected as follows:

$$
A\left(Z_{\text {eff }}\right)=1.9745 \cdot 10^{-31} Z_{\text {eff }}
$$

Page 171, first line above Equation(20.8):

and on the surface $\hat{q}=1$, Then $D_{0}=j_{0} q_{0} \beta$..

Page 172, the equation (20.11) should be corrected as follows:

$$
n_{e} \chi_{\text {eff }}=\frac{3}{2} \frac{E_{\text {ext }}^{2}}{A\left(Z_{\text {eff }}\right)} T^{1 / 2} \frac{h(r)}{\mu^{2}}=\frac{3}{2} \frac{E_{e x t} j(r)}{T} \frac{h(r)}{\mu^{2}},
$$

Page 182, after eqs.(22.6) and (22.8):

$k$-td, $j$-td should be replaced by $k$-th, $j$-th.

Page 183, the equation (22.10) should be corrected as follows:

$$
m \frac{d \vec{V}_{k}(t)}{d t}=q\left(\frac{1}{c} \vec{V}_{k} \times \vec{B}\left(\vec{x}_{k}, t\right)+\vec{E}\left(\vec{x}_{k}, t\right)\right)+\lambda \frac{q}{2}\left(\frac{1}{c} u_{j} \times \vec{B}\left(\vec{X}_{j}, t\right)+\vec{E}\left(\vec{X}_{j}, t\right)\right)
$$

Page 184, the first and the second line of section 23 should be corrected as follows:

In this section we assume that the velocity distribution of the particles is Maxwellian with local temperature $T_{j}$ in each $\Delta V_{j} \ldots$

Page 184, last line:

...isolated and the average $\overline{\overrightarrow{\tilde{V}}}_{j}$ vanishes..

Page 185, equation (23.8) should be corrected as follows:

$$
\overline{\overrightarrow{\tilde{V}}}_{j}=\frac{1}{N_{j}} \sum_{n=1}^{N_{j}} \int \overrightarrow{\tilde{v}} f d^{3} \tilde{v}_{1} \ldots d^{3} \tilde{v}_{N_{j}}=-\vec{v}\left(\vec{X}_{j}\right)+\left(\frac{\mu^{2} c}{4 \pi n q}\right) \vec{A}_{p}\left(\vec{X}_{j}\right)
$$


Page 191, second line:

.., and then $\int_{t_{1}}^{t_{2}} \delta L(t) d t=0$ follows..

Page 193: “(25.90” should be replaced by “(25.9)”.

Page 195: first line of Equation(26.6):

Replace $\frac{\varphi_{S}}{4 \pi} \int_{\partial \Omega} \delta \vec{E} \cdot d \vec{S}$ by $\frac{\varphi_{S} k_{m}^{2}}{4 \pi} \int_{\alpha 2} \delta \vec{E} \cdot d \vec{S}$

Page 214. the big dot in the exponential of Equation(33.7) should be replaced by the small dot: $\exp \left(-i \vec{k} \cdot\left(\vec{X}_{j}-\vec{X}_{j^{\prime}}\right)\right)$

\section{References and Notes}

1. Minardi, E. Thermodynamics of High Temperature Plasmas. Entropy 2009, 11, 124-221.

(C) 2009 by the authors; licensee Molecular Diversity Preservation International, Basel, Switzerland. This article is an open-access article distributed under the terms and conditions of the Creative Commons Attribution license (http://creativecommons.org/licenses/by/3.0/). 